\title{
The plasticity of the superior longitudinal fasciculus as a function of musical expertise: a diffusion tensor imaging study
}

\author{
'Department of Neuropsychology, Division of Psychology, University of Zurich, Zurich, Switzerland \\ 2 MR-Center, University Children's Hospital of Zurich, Zurich, Switzerland \\ ${ }^{3}$ Center for Integrative Human Physiology, University of Zurich, Zurich, Switzerland
}

Mathias S. Oechslin ${ }^{1,+}$, Adrian Imfeld ${ }^{1,+}$, Thomas Loenneker $^{2,3}$, Martin Meyer $^{1}$ and Lutz Jäncke ${ }^{1}$

\section{Edited by:}

Kenneth Hugdahl, University of

Bergen, Norway

\section{Reviewed by:}

Karsten Specht, University of Bergen, Norway

Mireille Besson, Institut de

Neurosciences Cognitives de la

Mediterranee, France

${ }^{*}$ Correspondence:

Mathias S. Oechslin, Department of

Neuropsychology, Division of

Psychology, University of Zurich,

Switzerland.

e-mail:m.oechslin@psychologie.uzh.ch

${ }^{+}$Mathias S. Oechslin and Adrian Imfeld have contributed equally to this work.
Previous neuroimaging studies have demonstrated that musical expertise leads to functional alterations in language processing. We utilized diffusion tensor imaging (DTI) to investigate white matter plasticity in musicians with absolute pitch (AP), relative pitch and non-musicians. Using DTI, we analysed the fractional anisotropy (FA) of the superior longitudinal fasciculus (SLF), which is considered the most primary pathway for processing and production of speech and music. In association with different levels of musical expertise, we found that AP is characterized by a greater left than right asymmetry of FA in core fibres of the SLF. A voxel-based analysis revealed three clusters within the left hemisphere SLF that showed significant positive correlations with error rates only for AP-musicians in an AP-test, but not for musicians without AP. We therefore conclude that the SLF architecture in AP musicians is related to AP acuity. In order to reconcile our observations with general aspects of development of fibre bundles, we introduce the Pioneer Axon Thesis, a theoretical approach to formalize axonal arrangements of major white matter pathways.

Keywords: DTI, absolute pitch, plasticity, musical expertise, superior longitudinal fasciculus, white matter, language, pioneer axon thesis

\section{INTRODUCTION}

The musician's brain has attracted much attention in recent years of neuroscience research as a model for brain plasticity (Münte et al., 2002). This research has shown that musicians demonstrate specific anatomical features related to their specific training expertise. In particular, those brain areas involved in controlling music-specific behaviour (auditory perception, motor control, and memory processes) reveal anatomical differences compared with non-musicians (NM). For example, there are differences between musicians and NM in volume and grey matter density in the auditory cortex, including Heschl's gyrus and the planum temporale (Schlaug et al., 1995a; Keenan et al., 2001; Schneider et al., 2002, 2005, 2009; Lüders et al., 2004; Hänggi et al., 2008; Bermudez et al., 2009). In addition to the auditory cortex, there are differences in Broca's area, the motor cortex, the cerebellum and parts of the parietal cortex (amongst others) between musicians and NM (Elbert et al., 1995; Schlaug et al., 1995b; Sluming et al., 2002, 2007; Gaser and Schlaug, 2003; Hutchinson et al., 2003; Bangert et al., 2006).

Besides these anatomical differences, numerous studies have shown that musicians process auditory and motor information differently, as indicated by neurophysiological measures (Pantev et al., 1998; Jäncke et al., 2000, 2006; Münte et al., 2002; Lotze et al., 2003; Fujioka et al., 2004, 2005; Peretz and Zatorre, 2005; Shahin et al., 2005; Koeneke et al., 2006; Baumann et al., 2007, 2008; Lappe et al., 2008; Herholz et al., 2009; Oechslin et al., 2010). Most of these studies have attributed these effects to the daily hours of intensive training over many years. Since most of these studies are cross-sectional in nature it is difficult to attribute these differences directly to music practice. However, some studies directly support the idea of practice-dependent effects in musicians. For example, Pantev and colleagues have shown that training can be instrument-specific (Pantev et al., 2001; Schulz et al., 2003; Shahin et al., 2008), and that the electroencephalography (EEG) responses of children taking music lessons change differently over the course of a year compared with those of children not studying music (Fujioka et al., 2006). A further finding is that musicians who have improved their auditory functions show also improved language functions as well as more efficient processing of language (Besson et al., 2007; Marques et al., 2007; Imfeld et al., 2009; Moreno et al., 2009; Oechslin et al., 2010).

In this study, we focus on a specific subgroup of musicians, namely those with absolute pitch (AP). In general, AP is defined as the ability to detect a pitch without any tonal reference. But it is still a matter of debate whether the extraordinary hearing acuity of AP develops due to musical training or is genetically determined (Vitouch, 2003; Levitin and Rogers, 2005). Regarding the neural underpinnings of AP, it has been shown that AP is characterized by specific features, mostly related to anatomical and physiological features and the specific role of the left hemisphere and left-sided perisylvian brain areas in particular. For example, a left-hemispheric volumetric asymmetry in the planum temporale (Schlaug et al., 1995a; Keenan et al., 2001; Gaser and Schlaug, 2003) has been reported. In terms of cortical activations, various studies demonstrated hemodynamic and electrophysiological lateralization effects (mostly favouring the left hemisphere) in musicians with AP while processing both music (Ohnishi et al., 2001; Schön et al., 2004; Gaab et al., 2006) and speech stimuli (Oechslin et al., 2010).

In the present study, we explored the anatomical characteristics of a particular fibre system, namely the superior longitudinal fibre tract (SLF), in AP and non-AP musicians [also called relative 
pitch (RP) possessors]. The SLF of particular interest because it connects the temporal areas involved in lower and higher order auditory processing with frontal brain areas involved in the control of several executive functions (including working memory, attention and many more) (Geschwind, 1970; Hagmann et al., 2006a). Evidently, the coordinated interplay between temporal and frontal brain areas is important for language and music functions (Aboitiz and Garcia, 1997, 2009; Glasser and Rilling, 2008; Rilling et al., 2008; Friederici, 2009). Thus it has been hypothesized that the evolution of this fibre tract is pivotal for the control of many cognitive functions, which are typical for human beings (e.g. language).

The SLF can be characterized as a bundle of white matter tracts including three distinct pathways: firstly, an anterior indirect segment connecting parietal cortical fields (inferior parietal lobe) with the frontal lobe (frontal operculum); second, a posterior indirect segment, which connects the perisylvian [posterior superior temporal gyrus (STG)], the middle and inferior temporal region with the parietal lobe; and, third, a long direct segment connecting the temporal lobe with the frontal lobe (Catani and Mesulam, 2008). In addition, the large bundle of fibres connecting the posterior part of the temporoparietal junction with the frontal lobe, called arcuate fasciculus (AF), includes sections of the two frontal projecting SLF pathways. Thus, the AF is considered to be part of the SLF. To date, several studies, each pursuing a different strategy of diffusion tensor imaging (DTI) data and fibre tractography (FT) analysis, have succeeded in tracing and separating SLF pathways (Makris et al., 2005, 2009; Barrick et al., 2007; Catani et al., 2007; Frey et al., 2008; Upadhyay et al., 2008).

DTI is the current standard for in vivo study of white matter anatomy of the human or animal brain and has attracted increasing attention over the past decade. While standard imaging-based neuropsychological research focuses on function and anatomy of grey matter using functional magnetic resonance imaging (fMRI) or voxel-based morphometry (VBM), the in vivo neuroimaging technique of DTI combined with fibre tracking (FT) offers a complementary way of exploring the architecture of white matter and axonal connectivity. DTI is based on magnetic resonance (MR) technology and provides measures of water diffusion in different spatial directions in the brain (Hagmann et al., 2006b; Mori and Zhang, 2006). The most commonly studied diffusion parameter is fractional anisotropy (FA), which quantifies the directionality of diffusion within a voxel between 0 (undirected, isotropic) and 1 (directed, anisotropic and is derived from the diffusion tensor (Hagmann et al., 2006b; Mori and Zhang, 2006). Since white matter in the brain consists of aligned axonal fibres, diffusion is constrained perpendicular to the orientation of these fibre bundles, which leads to anisotropic diffusion. The principal direction of diffusion reflects the orientation of a fibre bundle in a specific voxel and is, therefore, exploited by fibre tracking algorithms. The mean diffusivity (the trace of the diffusion tensor) is a measure for the amount of diffusion, which can be divided into an axial diffusivity component $\left(\lambda_{||}\right.$, diffusion along the axons) and a radial diffusivity component ( $\lambda_{\perp}$, diffusivity perpendicular to the axons). While $\lambda_{||}$corresponds to the first eigenvalue of the diffusion tensor $\left(\lambda_{||}=\lambda_{1}\right), \lambda_{\perp}$ is calculated by averaging the second and third eigenvalue: $\lambda_{\perp}=\left(\lambda_{2}+\lambda_{3}\right) / 2$ (Alexander et al., 2007).
FA has been found to increase during white matter maturation in the developing brain (Beaulieu, 2002; Cascio et al., 2007; Eluvathingal et al., 2007) and to decrease in the elderly (Moseley, 2002; Bhagat and Beaulieu, 2004). Reduced FA values have been reported in patients suffering from neurodegenerative diseases (Sundgren et al., 2004) or spinal cord injury (Wrigley et al., 2009). Furthermore, FA has been found to be positively correlated with behavioural measures such as reading ability (Klingberg et al., 2000; Beaulieu et al., 2005; Niogi and McCandliss, 2006), performance in a speeded lexical decision task (Gold et al., 2007), and musical sensorimotor practice (Bengtsson et al., 2005). However the relations between white matter development, degeneration and training-induced plastic changes on the one hand and water diffusion characteristics on the other still remain poorly understood and a matter of controversy (Beaulieu, 2002; Alexander et al., 2007; Ashtari et al., 2007). Notably only a small number of studies have tried to investigate the relationship between musical expertise, language associated cortical regions and related white matter structures using the DTI method, although it lends itself well to visualizing characteristic aspects of white matter plasticity as a function of musical practice and extensive hearing experience: So far differences have been characterized by greater values of FA in the genu of the corpus callosum, lower FA in the corona radiata and the bilateral internal capsule, respectively (Schmithorst and Wilke, 2002). Another study, particularly focusing on the corticospinal tract (CST), confirmed significantly lower FA values in both hemispheres of musicians compared to NM, whereas diffusivity in the CST was negatively correlated with the onset of musical training in childhood (Imfeld et al., 2009). Additionally, one DTI study identified positive correlations between the magnitude of local FA values and practice time in childhood and later life of professional musicians: bilaterally in the internal capsule, both in the splenium and the isthmus of the corpus callosum and in the ventral pathway of the right SLF (Bengtsson et al., 2005).

The above-mentioned studies confirmed white matter structurefunction correspondence related to long-term musical training. Recent studies using fMRI and EEG provide strong evidence for alterations not only in music but also in speech processing modulated by musical expertise (Schön et al., 2004; Marques et al., 2007; Musacchia et al., 2007; Catani and Mesulam, 2008; Oechslin et al., 2010). In this context, it would therefore be promising to focus on the white matter structure, which is responsible for a proper neural information supply in order to guarantee an accurate decoding of acoustic signals and speech production, respectively.

Several studies have shown that AP is related to processes and anatomical grey matter differences, which are lateralized to the left perisylvian brain area (Schlaug et al., 1995b; Ohnishi et al., 2001). The present study aimed to elucidate the extent to which white matter differences of the SLF reflect functional and anatomical lateralization effects in association with the acuity of AP.

\section{MATERIALS AND METHODS PARTICIPANTS AND ABSOLUTE PITCH TEST}

A total number of 39 subjects participated in this study: 13 professional musicians with AP, 13 professional musicians with RP, and $13 \mathrm{NM}$ as control subjects. All musicians had started musical practice before the age of 10 (Table 1). All participants were 
Table 1 |This table displays group specific data of gender, age, practice start, total amount of practice years and errors in the AP-test (mean \pm SD). None of the parameters differs significantly with respect to the observed experimental groups except the evaluated AP performance [one-tailed $t$-test for independent samples: $p<0.001(t=18.75)$, Kolmogorov-Smirnov test demonstrated normally distributed AP-test performance in RPs and APs $(p>0.2)$.

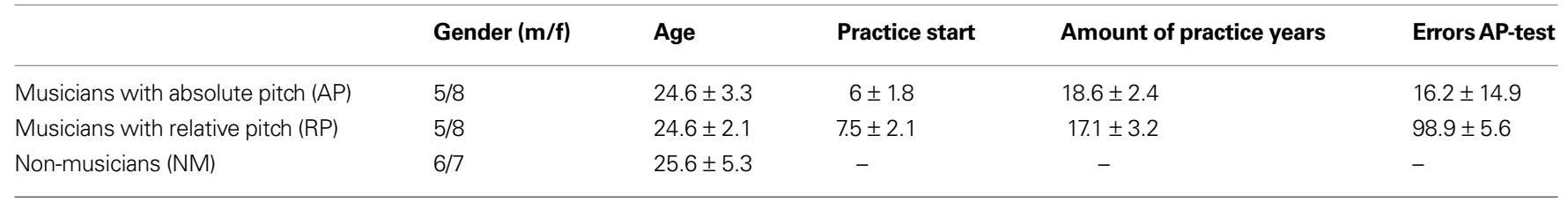

tested for their hand preference using the Annett Handedness Questionnaire (Annett, 1967) and were verified according to the procedure described by Annett as consistent right-handers. The musicians played a variety of instruments including the violin, viola, piano, trombone and the traverse flute. Local ethical committee approval was obtained and all subjects provided written informed consent consistent with the Declaration of Helsinki.

We evaluated AP among professional musicians (AP and RP) with an in-house designed test: participants heard 108 pure sine wave tones, presented in pseudo-randomized order, which ranged from $\mathrm{A} 3$ (tuning: $\mathrm{A} 4=440 \mathrm{~Hz}$ ) to $\mathrm{A} 5$, while each tone was presented ninefold (threefold in each octave). The accuracy was evaluated by counting correct answers - the semi-tone errors were taken as incorrect to increase the discriminatory power by means of AP. Furthermore, the participants were not asked to identify the adjacent octaves of the presented tones, as for AP it is a most notable prerequisite to identify the correct chroma. Accurate detection of octaves is quite a difficult task, which is hardly possible even for musicians with AP.

Each tone of the AP-test had duration of $1 \mathrm{~s}$; the inter-stimulus interval (ISI) of $4 \mathrm{~s}$ was filled with brown noise. Subjects had to write down the tonal label immediately after they heard the accordant tone (i.e. while hearing the $4 \mathrm{~s}$ of brown noise).

\section{DTI DATA ACQUISITION}

We performed DTI on a 3T whole body MR system (Signa Excite II, GE Healthcare, Milwaukee, USA) using a standard 8-channel head coil. Axial imaging was performed in accordance with an imaging plane parallel to the anterior-posterior commissural (AC-PC) line. We sampled the diffusion tensor by repeating a diffusion-weighted single-shot spin-echo echo-planar sequence along 21 different geometrical directions. Diffusion sensitization was achieved with two balanced diffusion gradients centred on the $180^{\circ}$ radio-frequency pulse. An effective $b$-value of $1000 \mathrm{~s} / \mathrm{mm}^{2}$ was used for each of the 21 diffusion-encoding directions. Three measurements were performed without diffusion weighting $\left(b_{0}\right.$ of $\left.0 \mathrm{~s} / \mathrm{mm}^{2}\right)$ at the beginning of the sequence. Scan parameters were TR $=8000 \mathrm{~ms}$; $\mathrm{TE}=91 \mathrm{~ms}$; matrix size $=128 \times 128$; and FOV $=240 \times 240 \mathrm{~mm}$. A total of 42 contiguous 3-mm-thick axial sections were acquired. Data quality was examined using DTI-Studio (Jiang et al., 2006). And bad slices were excluded from analysis (11 bad slices out of all acquired slices $(39 \times 24 \times 42=39312)$ in the three groups $(\mathrm{AP} /$ $\mathrm{RP} / \mathrm{NM}$ ) of participants).

\section{PREPROCESSING}

FA and mean diffusivity (trace) images were calculated in native space using DTI-Studio (Jiang et al., 2006). Preprocessing of these images consisted of four steps: (1) brain extraction, (2) nonlinear normalization to Montreal Neurological Institute (MNI) space, (3) smoothing for voxel-wise analysis, and (4) thresholding for selection of white matter only.

Normalization was done by estimating the morphing parameters from a nonlinear normalization of the mean $b_{0}$ image's grey matter component to SPM5's grey matter probability map. These parameters where then applied to the FA and diffusivity images, resulting in accurate alignment of the SLF in all subjects. The smoothing kernel of $8 \mathrm{~mm}$ was chosen according to standards in DTI literature (Snook et al., 2007). However, for ROI analysis we used unsmoothed data (voxel size $2 \times 2 \times 2 \mathrm{~mm}$ ) to avoid partial volume contribution by signal from neighbouring non-white matter voxels. Thresholding for selection of white matter only was done by subjecting FA and diffusivity images to a combined threshold of FA $>0.25$ and trace $<0.004$.

\section{FIBRE TRACTOGRAPHY}

In order to determine regions for ROI analysis, SLF probability maps for each group and each hemisphere were created, using deterministic FT. First, diffusion tensors were calculated in MNI stereotaxic standard space for every subject using the diffusion II toolbox for SPM available at htttp://souceforge.net/projects/spmtools. The tensor data were transformed into a data format amenable to the DTI\&Fibre Tools software package for fibre tracking and visualization (Kreher et al., 2006). Using this software, a deterministic fibre-tracking algorithm (Mori et al., 1999) was performed on the whole brain, initiating a fibre at the centre of every voxel. Tracking was automatically terminated if the FA value of the current voxel dropped below 0.15 or mean diffusivity exceeded 0.002 , indicating grey matter or cerebro-spinal fluid voxels. Fibres were also terminated if the angle between the principal eigenvectors of the current and the next voxel exceeded 53.1 ${ }^{\circ}$ (Kreher et al., 2006).

According to the described fibre tracking strategy by Mori et al. (2002) a multi-ROI approach in MNI space was used to select the SLF fibre structure. As depicted in Figure 1 we used two spatially separated ROIs to reconstruct the whole SLF pathway (Catani et al., 2007) - this procedure covers both the indirect (parietal-frontal-projection) and direct pathway (temporo-parieto-frontal projection).

Since fibre tracking was performed in MNI space, the process of extracting the individual fibre structures of each subject was automated in order to reduce observer bias. The resulting fibre structures were visually inspected and only an ignorable number of spurious fibres not belonging to the SLF had to be eliminated manually.

Visit masks (ROIs marking voxels which contain fibres of a specific fibre tract) were generated for the SLF fibre structure in the two hemispheres of every subject. These binary visit-masks 
were averaged across subjects (13 subjects per group) in order to obtain group probability maps for each hemisphere as shown in Figure 2.

\section{ROI ANALYSIS BASED ON THRESHOLDED SLF PROBABILITY MAPS}

Group-specific ROIs for the left and the right SLF were derived from the probability maps by applying a threshold at $p<0.05$, to obtain volumetric regions with a maximized probability of fibre trajectories. Thus we extracted volumetric ROIs of asymmetric shapes that represent group and hemisphere specific fibre volumes in voxels (Figure 3). This approach allows registration of subtle differences of SLF volumes between groups and hemispheres that would be washed out when using a standard approach. Extraction of binary ROIs from probability maps was used within SPM5 (http://www. fil.ion.ucl.ac.uk/spm/software/spm5/). Mean FA-values were extracted from the ROIs of all 39 subjects using an in-house written Matlab script. Two-way ANOVA (hemisphere $\times$ expertise) with one repeated measurements factor (hemisphere) and subsequent two-tailed paired samples $t$-tests (Bonferroni corrected for multiple comparisons) and was performed to evaluate hemispheric differences as a function of musical expertise.

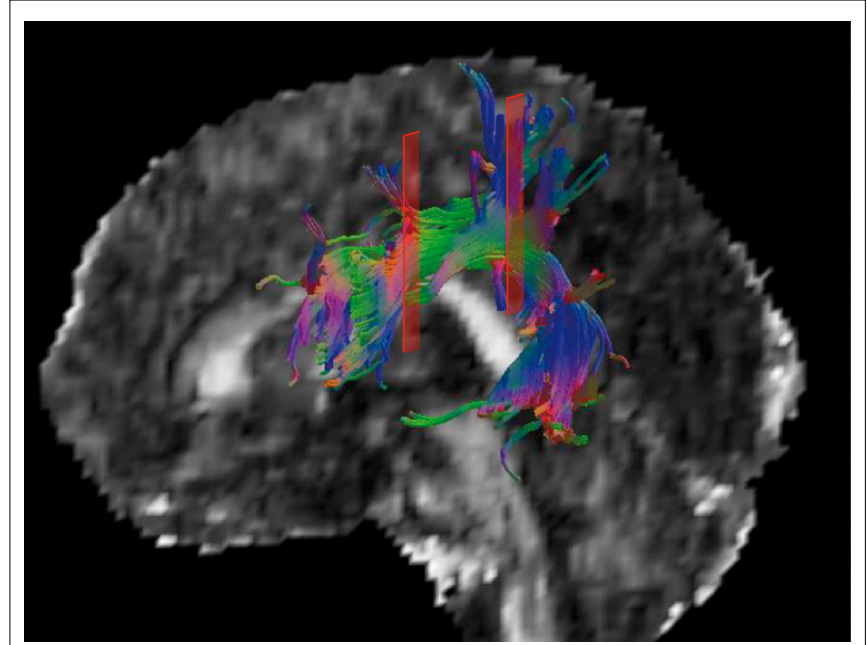

FIGURE 1 |A 3-D model of the left SLF of a single subject is displayed. The red areas indicate the anterior and posterior ROI used for creating the SLF fibre subset. Only fibres running through both $\mathrm{ROIs}$ are considered to be part of the tracked fibre structure.

\section{VOXEL-WISE ANALYSIS OF MUSICIANS' SLF}

To examine the relation between local anisotropic characteristics and AP-test performance, we performed multiple regression within SPM5. To focus on the SLF structure bilaterally this voxel-wise analysis, we used the AP group-specific unthresholded probability map as binary template for bilateral explicit masking (Figure 4). Subsequently, significant clusters $[p<0.01$ (unc.), $k=5]$ were extracted using the SPM5 toolbox marsbar, and served as ROIs for the analysis of individual FA maps. Although one might be dubious about the application of cluster ROIs instead of more established spherical ROIs, we prefer to apply the former in this case, since this strategy allows the most precise determination with respect to the SLF structure (defined by the SLF binary mask) and white matter in general. This procedure has been executed in both groups of musicians (AP and RP), but not in NM, since the latter group is not able to perform the AP-test due to musical illiteracy. Furthermore, we correlated mean FA values with the AP-test errors of the accordant group by calculating the Pearson's correlation coefficients (Figure 5).

\section{RESULTS}

The SLF delineated on the basis of the probability maps is shown in Figure 3. Based on six a priori defined ROIs ( 3 groups $\times 2$ hemispheres) the mean FA values were subjected to a two-way ANOVA [expertise $(\mathrm{AP} / \mathrm{RP} / \mathrm{NM}) \times$ hemisphere $(\mathrm{LH} / \mathrm{RH})]$ with repeated measurements on one factor (hemisphere) and revealed significantly between-hemisphere differences as a function of musical expertise [interaction expertise $\times$ hemisphere: $F(2,36)=7.04, p<0.01$ ]. In order to qualify this interaction we conducted subsequent post-hoc paired $t$-tests $(p<0.05$, Bonferroni corrected) and identified that AP musicians showed a significant left-greater-than-right lateralization $(t=2.8$, $p=0.016)$. NM on the other hand showed a significant right-greaterthan-left lateralization $(t=-2.79, p=0.016)$, whereas RP showed no inter-hemispheric differences $(t=-0.63, p=0.543$; Table 2).

Multiple regression of APs' FA maps using their AP-test error rates as covariates revealed three clusters located exclusively within the left-hemispheric SLF indicating significant positive correlations between error rates and FA indices of APs (Figure 5A). In other words, high performance in the AP-test is associated with low mean FA values within the observed regions. These clusters are situated in portions of the SLF which connect anterior, posterior and perisylvian regions that are essential for the cortical organization of speech processing (Hickok and Poeppel, 2007): The first one, representing the cluster with the highest correlation effect $(-28,-28$,

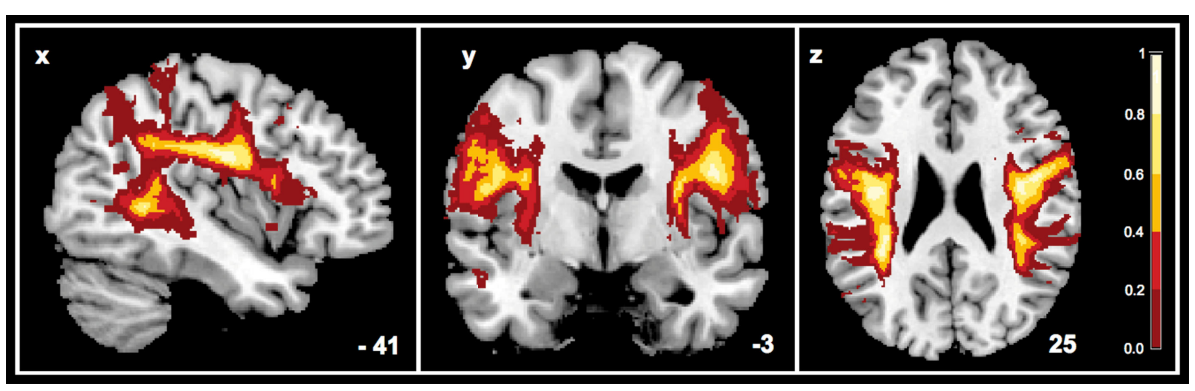

FIGURE 2 | Here the probability map for the SLF of the AP group $(n=13)$ is illustrated - combined for two hemispheres. Voxels are displayed with unthresholded probabilities. 

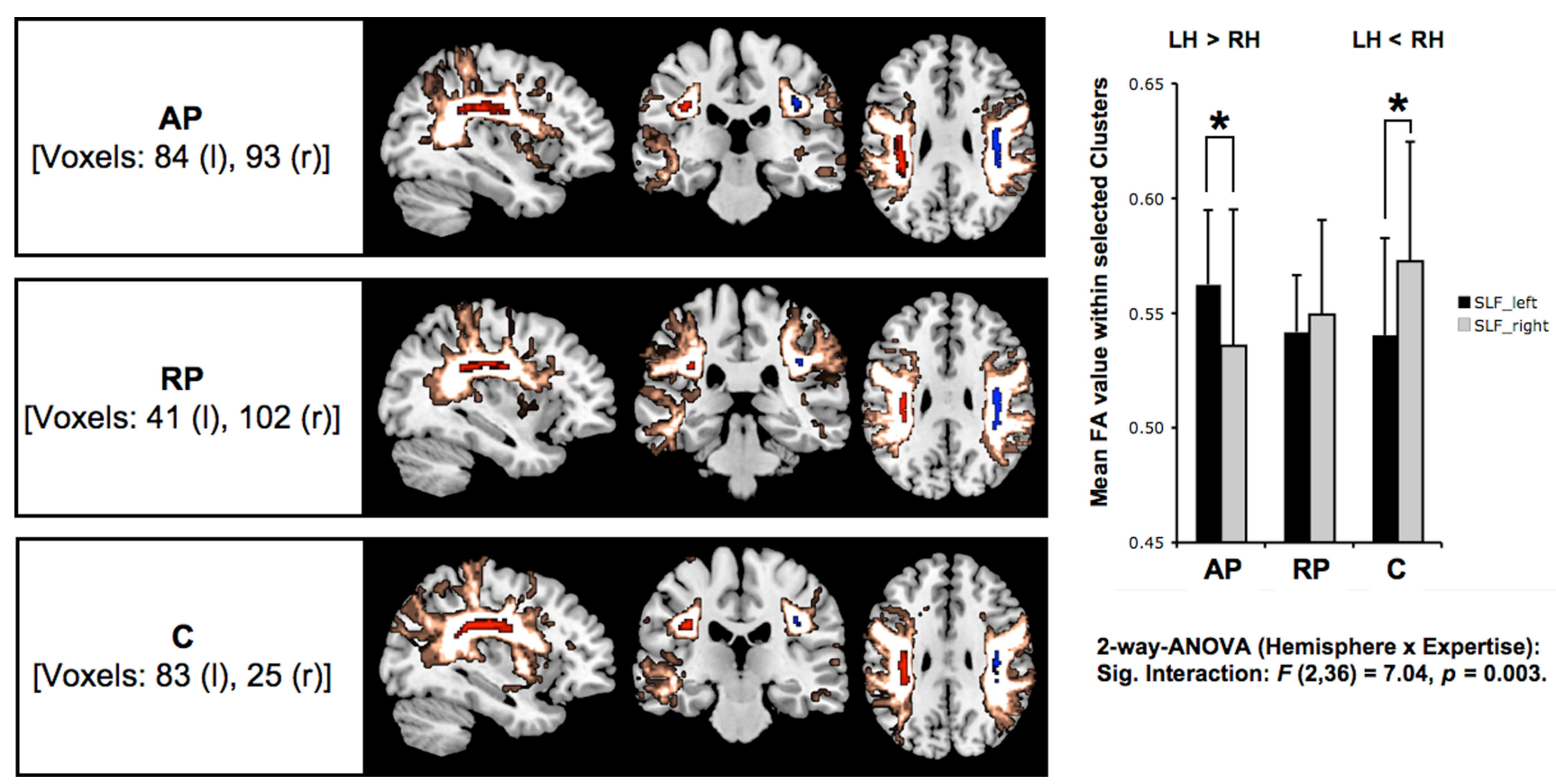

FIGURE 3 |The ROIs calculated by thresholding probability maps of bilateral SLFs' are illustrated in red (left) and blue (right), surrounded by unthresholded group specific probability maps (gold). The bar chart illustrates for each group and both hemispheres the standard deviation (error bars) and means of FA values calculated by comprising all suprathreshold voxels within the defined ROls $(p<0.05)$. Asterisks indicate significant inter-hemispheric within-group differences $\left({ }^{*} p<0.05\right)$ as a result of $t$-tests for paired samples (two-tailed). Moreover, the between-group analysis (two-way ANOVA) revealed a significant interaction expertise $\times$ hemisphere.

2-way-ANOVA (Hemisphere $x$ Expertise): Sig. Interaction: $F(2,36)=7.04, p=0.003$.

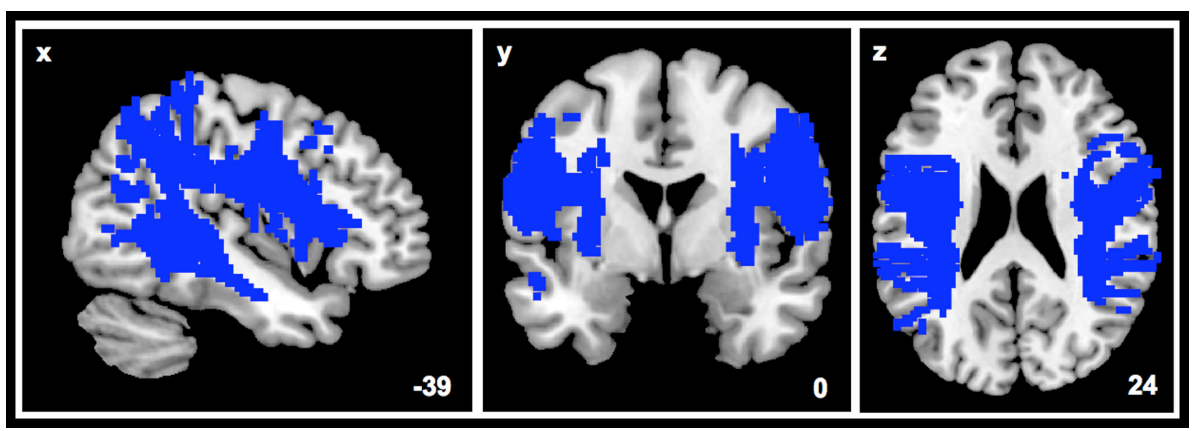

FIGURE 4 |The unthresholded probability map of the bilateral SLF served as template to create the illustrated binary mask while performing the voxelwise analysis.

$44 ; k=6, t=4.15, p=0.01$, uncorrected; Figure 5B) is located at the superior border of the frontoparietal SLF projection, next to the fundus of the postcentral gyrus; the second is located deeply within the inferior-frontal ramifications projecting into the frontal operculum $(-40,-2,22 ; k=9, t=3.16, p=0.01$, uncorrected; Figure 5C); and the third most extended cluster is located laterally at the SLF's posterior indirect segment projecting to the inferior temporal sulcus and the middle temporal gyrus (MTG) $(-42,-50$, $4 ; k=20, t=3.94, p=0.01$, uncorrected; Figure 5D).

Based on the ROI analysis, as described in Section "Materials and Methods," and the correlation of mean FA indices of the cluster ROIs with the AP's error rates, we emphasize the relationship indicated by voxel-wise analysis: In relation to the auditory acuity of AP, all clusters revealed significant $(p<0.01)$ positive correlations $(r)$ between 0.69 and $0.77(n=13)$, whereas in RP there is no evidence for a relationship between their error rates and FA values for the same sample of ROIs (Figure 5).

\section{DISCUSSION}

This study focused on alterations in white matter as a function of musical training and exceptional hearing abilities. Our specific interest, as outlined in the introduction and reflected in our analysis, concerned whether and to what extent there are differences in FA-values within the SLF structure between (1) musicians and NM, and (2) between musicians with and without AP. Firstly, we discuss ROI-based effects of hemispheric lateralizations that reflect 

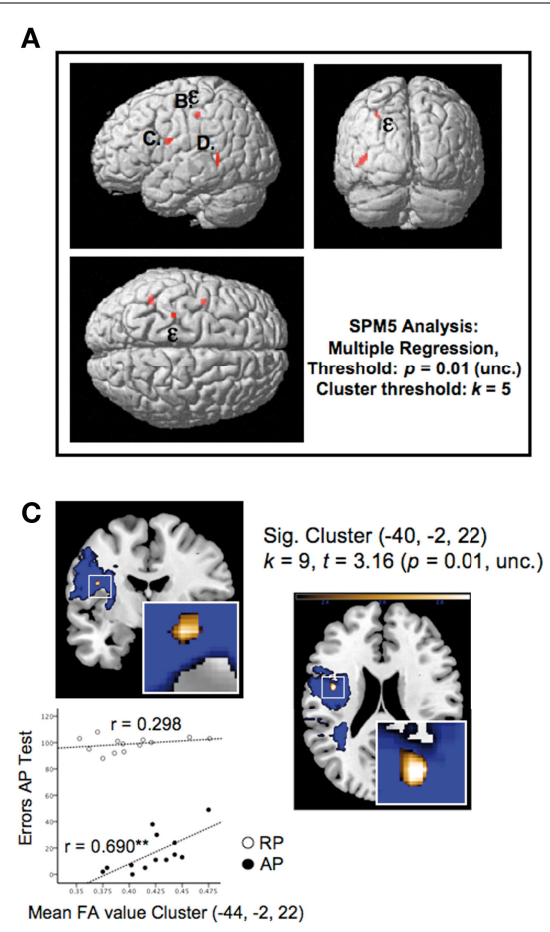

FIGURE 5 | (A) Shows the three significant clusters (B-D) identified by a voxel-wise multiple regression, characterized by FA values which significantly correlate with the error rates of the APs in the AP-test. The highest correlational effect [designated by the epsilon $(\varepsilon)$ ] has been identified as the most superior located cluster (B) of the SLF. (B-D) provides close inspections of the significant clusters and plotted correlations obtained by the additional ROI analysis, which is based on the identified clusters in APs (see
B

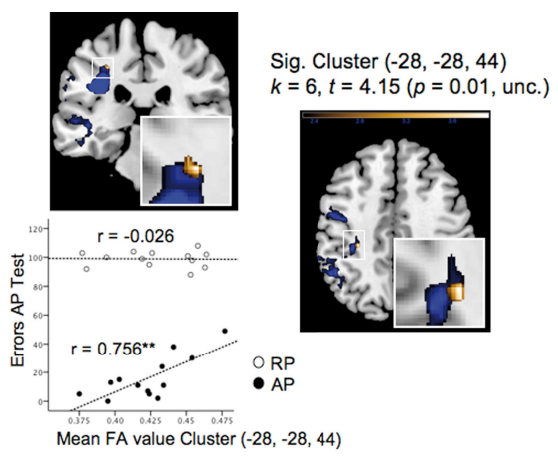

D

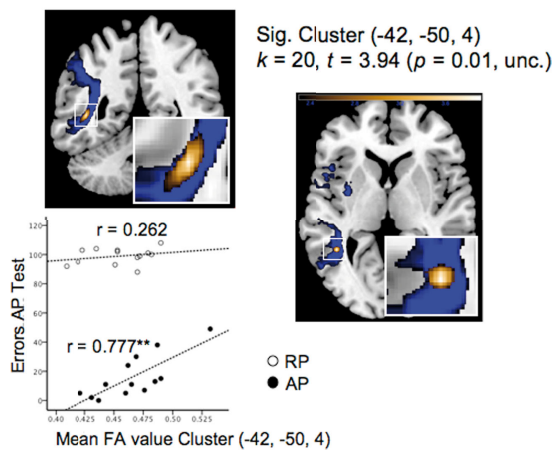

Section "Materials and Methods"). Accordingly, the error rates are plotted against mean FA values of RP and AP found by the ROI analysis. Asterisks $\left({ }^{*} p<0.01\right)$ indicate the level of significance with respect to the calculated correlational coefficients (Pearson's 1 ). Furthermore, (B-D) provides additional information concerning the corresponding cluster $[k=$ number of voxels, $t=$ highest $t$-value within the cluster, $(x, y, z)=$ coordinates of the peak voxel of each cluster in MNI space].
Table 2 |The upper part of this table contains the descriptive data (mean $\pm \mathrm{SD}$ ) reflecting the performed $\mathrm{ROI}$ analysis based on thresholded probability maps. Additionally, the t-tests ( $\left.{ }^{*} p<0.05\right)$ show within-group differences (Bonferroni corrected) of the mean FA values between the left $(\mathrm{LH})$ and the right hemisphere $(\mathrm{RH})$.

\begin{tabular}{llll}
\hline & AP & RP & NM \\
\hline FA left SLF (mean \pm SD) & $0.562 \pm 0.04$ & $0.542 \pm 0.05$ & $0.540 \pm 0.05$ \\
FA right SLF (mean \pm SD) & $0.536 \pm 0.05$ & $0.549 \pm 0.03$ & $0.573 \pm 0.06$ \\
\multicolumn{1}{l}{-Tests (paired, two-tailed) LH vs. RH } & & \\
$t$ & 2.8 & -0.63 & -2.79 \\
$p$ & $0.016^{*}$ & 0.543 (n.s.) & $0.016^{*}$ \\
\hline
\end{tabular}

between-group differences. Secondly, our discussion emphasizes AP-specific FA characteristics for the SLF as a whole. Finally, we discuss our results in the context of myelination principles and axonal formation and we introduce the Pioneer Axon Thesis.

\section{ROI ANALYSIS DUE TO PROBABILITY MAPS}

The utilization of group-specific probability maps for ROI generation revealed a lateralization effect as a function of musical expertise (as revealed by the ANOVA). We found greater left than right lateralization of mean $\mathrm{FA}$ values in $\mathrm{AP}$ and greater right than left lateralization in NM, while there was no significant lateralization effect in RP. In contrast, the analysis of the complete structure of SLF, by averaging FA values of all corresponding voxels (Figure 4), revealed no significant lateralization effect as a function of musical expertise.

The group-specific lateralization of mean FA values (Figure 3) cannot be explained by differences in the biographic variables age, onset of musical training, or total amount of practice years (Table 1). The inclusion of these biographical parameters - both partial (corrected for age) and bivariate - in our correlational approach allows us to rule out any significant relationship between these demographic parameters ${ }^{1}$ and mean FA values within the ROIs of the left/right hemisphere in all three groups. A meaningful influence of these factors on the demonstrated group-specific lateralization effects appears unlikely. The significant interaction expertise $\times$ hemisphere and the results of post-hoc $t$-tests suggest that the SLF asymmetry is directly related to the acuity of AP and musical expertise.

The present data is in accordance with the literature pointing out the special role of the left hemispheric auditory processing by means of structural (Schlaug et al., 1995a; Keenan et al., 2001; Lüders et al., 2004) and functional plasticity (Pantev et al., 1998; Ohnishi et al., 2001; Oechslin et al., 2010) modulated by the level of musical exper-

${ }^{1}$ With one single exception: mean FA-values of C's left hemispheric SLF ROI are significantly correlated with age $\left(r=0.573^{\star}, p=0.041\right)$. 
tise and AP in particular: Generally speaking, the finding concerning the significant interaction hemisphere $\times$ expertise indicates substantial anatomical between-group differences in terms of measuring white matter properties of the SLF core structure irrespective of what we know about FA-values. However, taking into account that in principle relatively high anisotropy is associated with increased diffusivity (Hagmann et al., 2006b) when focusing on neural populations with a high degree of parallel aligned fibres (like in the core of the SLF) we can conclude, that AP musicians obviously show a stronger connectivity of axonal projections within the left SLF than in the right SLF, whereas NM show a vice-versa relationship and RP show no hemispheric difference. With respect to the above mentioned structural and functional hemispheric preponderance of auditory signal processing in musical experts, the present findings seem intriguingly to introduce a developmental specialization of the left SLF architecture by means of an enhanced fibre connectivity and thus information transfer in musicians with AP.

\section{MULTIPLE REGRESSION EFFECTS}

Our study sought to investigate AP-specific anisotropic distributions in the SLF structure, the results of the multiple regression revealing an interesting pattern. This comprised three left-lateralized clusters in which FA values correlated positively with the error rates of the AP-musicians such that low FA values correspond with high performances in the AP-test. That these clusters were located at important areas of the SLF architecture and of language processing (Glasser and Rilling, 2008) leads us to assume that this structural asymmetry might have functional implications.

We begin by discussing the two clusters that are part of the arcuate branch of the SLF (Figures 5B,C). One of these was located at the superior border of the frontoparietal connection (AF) and is conspicuous by its vicinity to the inferior parietal region, whereas the second was located deep inside the frontal arcuate bundle as it projects into the frontal operculum. Recent observations demonstrate that these latter two cortical grey matter regions are involved in action-related sound recognition (Lahav et al., 2007). In this study, these two regions were activated simultaneously when listening to a newly acquired piece of music, but only in NM. Of course, this function of audio-motor integration is one of the most important aspects of AP, both with respect to acquisition of AP and daily practice by AP-musicians. This rare group of musicians most likely associates any heard musical pitch with the corresponding finger position on the instrument. It is thus reasonable to assume that these two clusters are unique to musicians with AP, presumably reflecting AP's exceptional associative linkage between auditory and motor function.

The third SLF cluster was functionally associated with the posterior auditory cortex, and projects to the posterior STG, the superior temporal sulcus (STS), and the MTG (Figure 5D). Although evaluation of the exact fibre termination was not possible, the role in speech and auditory processing of the terminal zones of the SLF is undisputed (Buchsbaum et al., 2001; Vigneau et al., 2006). In favour of our interpretation, a recent DTI study identified two distinct SLF pathways in the left hemisphere projecting to language-related cortical areas that both connect the inferior-frontal area with the posterior perisylvian region and the middle-temporal region, (Glasser and Rilling, 2008). In this study, tractography results were compared with peak activation coordinates from prior functional imaging studies. In particular, the authors compared the volumes of the identified pathways between left and right hemisphere. Grey matter STG terminations of the SLF were found to be strongly left lateralized and associated with phonological activations in the left but not in the right hemisphere. Similarly, the MTG terminations were also found to be strongly left lateralized, overlapping in this case with activations associated with lexical semantic activations. Even if these findings are not specifically related to musical expertise, our data are well in line with a previous paper of our group (Oechslin et al., 2010) that reported modulation of activation in brain areas involved in higher language processing by musical expertise and AP. More specifically, musical expertise (AP in contrast to NM) was associated with functional alterations in the posterior parts of the left hemispheric MTG and STS. Given the consistency of this data with that of the current study, we suggest a relationship between local FA values and AP ability that reflects a strong link between musical expertise, AP, and language processing.

\section{NEURAL FORMATION AND AXONAL MYELINATION}

In the following, we specify the rationale behind our hypothesis that this lateralization effect is a core feature of AP and based on primal neural formation and myelination of the AF, as part of the SLF, possibly evident long before these individuals become aware of AP.

We now discuss this issue more comprehensively in order to subsequently put forward what we term the Pioneer Axon Thesis (Figure 6 depicts a schematic illustration of different possible organizational principles of the architecture of the arcuate fibre bundle). The principles of axonal guidance have been well investigated (Sperry, 1963; Tessier-Lavigne and Goodman, 1996; Yu and Bargmann, 2001; Guan and Rao, 2003). But the current lack of histological longitudinal studies of white matter development during critical periods of brain development means that, as far as we know, no studies have yet addressed the architectural fibre arrangement of white matter fasciculi in the human brain. But several DTI studies have focused on the development of white matter in different stages of maturation: in premature newborns (Partridge et al., 2004, 2005), during the first 2 years of life (Mukherjee et al.,

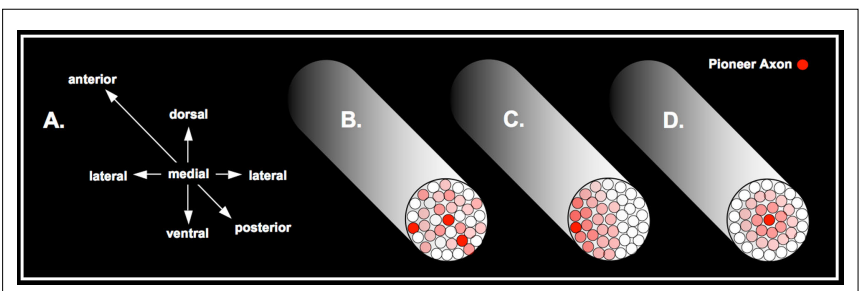

FIGURE 6 | Here a schematic cross-section of the arcuate fasciculus is delineated. On the left (A) the orientation of the fasciculus are defined by three axes anterior-posterior, dorsal-ventral and lateral-medial. The second to the left scheme (B) represents a chaotic distribution of axonal fibres, with several possible arbitrary positions of the pioneer axon, whereas the increased red colour typifies older axonal pathways. The second to the right scheme (C) stands for a possible alternative order driven by a laterally oriented axonal architecture. At the right scheme (D), the proposed architecture due to the maturation of the fasciculus is outlined as suggested by the Pioneer Axon Thesis - thus, the pioneer axon is located medially. 
2002; Schneider et al., 2004; Gao et al., 2009) and in later life and adolescence (Schmithorst et al., 2002; Snook et al., 2005, 2007). In investigating different DTI parameters like FA, radial and axial diffusivities, these studies provide important data on early maturation and myelination of different white matter structures in the developing brain. The study of cytological and quantitative aspects of callosal axon addition and elimination in rhesus monkey (LaMantia and Rakic, 1990) has shown axon addition occurring exclusively during the period of midgestation through to birth and elimination of about $70 \%$ of the callosal axons 4 months after birth. And, in a study that focused on the frontal and the temporal lobe in human brains, a linear degeneration of white matter volume that continued into the fifth decade of life was demonstrated (Bartzokis et al., 2001).

However, it is in postnatal life that reduced tortuosity towards a more straightened, parallel and dense fibre architecture as a function of aging emerges (Takahashi et al., 2000; Paus, 2005; Ashtari et al., 2007). Fibre orientations especially in the SLF have been revealed to be rather random in neonates and to show gradual organization along the anterior-posterior orientation during development (Zhang et al., 2007). Most importantly, of the language-related regions (involved in both perception and production), the AF matures last in terms of myelination (posterior sylvian region, Broca's area, primary motor cortex, primary visual cortex, primary auditory cortex and visual-auditory transition area - gyrus angularis). The pace of myelination of the AF in particular is reported to be similar to these language-related regions, but decelerates after 3 years of age (Su et al., 2008). This characteristic pattern of slow progression of the AF appears to be strongly linked to language acquisition and an age-related increase in vocabulary (Kuhl, 2004; Pujol et al., 2006; Zhang et al., 2007). This is in line with important findings that the associative parietal and temporal fasciculi are the last structures to commence myelination, showing a protracted course into adulthood (Paus et al., 1999; Dubois et al., 2008). In general, myelination processes actually affect diffusion characteristics that are considered to continue over a relatively long period of life: Using in vivo morphometry in 111 children (aged 4-17 years), the left AF was found to correlate positively between age and white matter density (Paus et al., 1999). According to these authors, the parameter density reflects increases in axon diameter, myelination or iron concentration, separately or in combination. Cross-section DTI studies of children and adolescents revealed that FA values are significantly positively correlated with age in the language-relevant structures STG, MTG and STS (Barnea-Goraly et al., 2005) and left AF (Schmithorst et al., 2002; Ashtari et al., 2007).

But there is to our knowledge no data on which basis the local fibre arrangement may be interpreted as an expression of cortical maturation within single white matter structures. We therefore propose the Pioneer Axon Thesis as a tentative approach for interpreting our DTI data within the context of axonal development and the organizational principles underlying the formation of major white matter pathways like the AF.

We propose a link between axonal arrangements and myelination processes: Since the axonal connections are terminated very early in postnatal life compared with long-lasting myelination processes, we assume that this developmental asynchronicity has a considerable impact on local anisotropic properties of white matter pathways; we assume that early-established, core axonal connections are characterized by diverging diffusion qualities compared with more distal pathway sites, which, according to the cited literature in the preceding, undergo plastic change over a long period of time into adult life.

In general, we suggest there may be a number of different axonal arrangements which may occur in the process of forming a white matter pathway: a random pattern of arrangement may occur in which the pioneer axon could be localized at any possible site within the fasciculus, as illustrated in Figure 6B. Figure 6C illustrates any possible lateral position of the pioneer axon, all other subsequently developed axons having a clear spatial relation. The manifest architecture is characterized by a consistently laterally oriented pattern of fibre maturation. Figure 6D depicts a fibre arrangement, which, from our point of view, is the most likely for a number of reasons outlined in the following. Here, the pioneer axon is located medially, whereas the subsequently formed axons build a concentric architecture as a function of maturation and time.

Given that an initial neural connection between two cortical regions is built up by a pioneer axon, subsequent guided axons will appear according to the relevance and frequency of an innervated connection. Even though future axon generations - ultimately making up a proper fasciculus - arrange themselves in a random pattern, it is reasonable to assume that these axons tend to maintain a certain proximity to the ancestral axons. It is conceivable that this tendency most probably ensures an efficient and preferentially targeted connection between two cortical regions (in case of the AF: the connection between inferior-frontal and temporoparietal/ temporal regions). It follows from this that spatial aspects play a major role in the final architecture. Irrespective of axon diameter, an arrangement has to be considered that allows a smooth coexistence of parallel-aligned axons especially in view of the reduction in disposable space over the protracted course of myelination. In summary, compared with earlier generations of axons later generations are forced to choose a trajectory that is radially more distant from the pioneer axon but still as close as possible. Consequently, these processes would yield a concentric architecture reflecting the developmental course of a white matter pathway as illustrated in Figure 6D.

In assuming that white matter expansion may occur as a function of learning history, we interpret our DTI data as follows in the context of the proposed mechanism of neural formation and myelination. The implication of this for musical expertise and particularly AP is that there may be a link between expertise and certain anisotropic alterations that may be distinguishable in location and characteristics from earlier (pre-expertise) developed parts of the SLF.

\section{INTEGRATION OF THE PIONEER AXON THESIS}

The fact that the ROIs (Figure 3 ) were created by thresholding the probability maps $(p<0.05)$ corroborates the suggestion that the identified ROIs represent ontogenetically earlier sections of the AF, but this does not apply for the localization on the anterior-posterior axis. This is because the six positions of the identified ROIs ( 3 groups $\times 2$ hemispheres, Figure 3 ) depend on the two intersectional ROIs that were used to track the SLF of single 
subjects (Figure 1), which in turn form the basis for creating probability maps. In this context, the essential role of DTI probability maps might be clearer by referring to their main attribute; because high probability for local (voxelwise) fibre occurrence is a consequence of low inter-individual variability of fibre presence. Since in the AF high inter-individual morphological variations are assumed to develop at later stages of cortical maturation, we suggest that structures with very low inter-individual variability and high morphological consistency tend to develop in very early maturational stages. This argumentation is supported by a longitudinal study of grey matter maturation that demonstrated a heterochronous pattern of cortical development (Gogtay et al., 2004): In general, the data lead the authors to suggest that phylogenetical older cortical areas mature earlier compared with more recent evolved cortical regions.

As the identified ROIs of SLF (Figure 3 ) represent the regions of lowest inter-individual variability, a plausible explanation is that ontogenetically early fibre tracts characterize the core section of a gross pathway like the AF. This conclusion is consistent with our Pioneer Axon Thesis, which presumes that the underlying architecture of a white matter pathway is determined by a concentric axonal arrangement that reflects the process of cortical maturation and the following spatio-temporal pattern of myelination.

In view of the local arrangement of both the identified ROI of APs left hemispheric SLF and the identified cluster (the latter representing voxels with a significant negative correlation between FA-values and AP-test error-rate), it is clear that the ROI (red coloured) is located at the core of the SLF, whereas the identified cluster (golden coloured, also in Figure 5B) is located at the boarder of the SLF (Figure 6).

We assume therefore that the group-specific lateralization effect (Figure 7, red coloured, core arcuate region) putatively reflects an earlier developmental stage before and during deliberate musical practice (pre-expertise-state). In addition, we identified by multiple regression a distinctive peripheral cluster (Figure 7, golden coloured, superior border arcuate region) which might be associated with plastic changes following later acquired musical expertise and AP. This would be consistent with a DTI study (McGraw

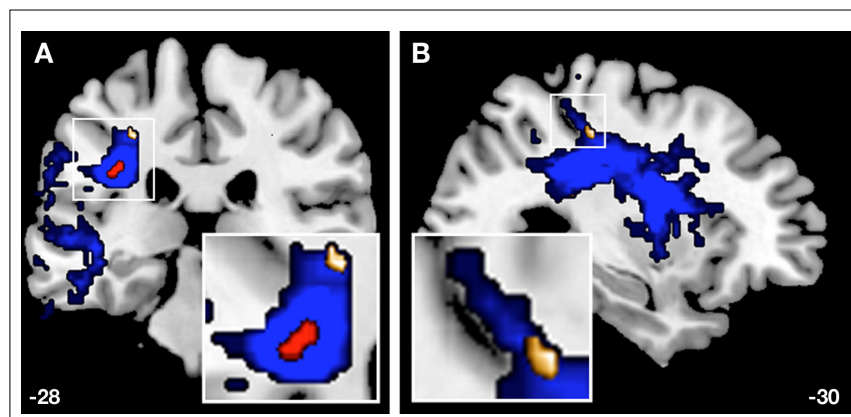

FIGURE 7 |A coronal (A) and a sagittal (B) view of the probability map of the APs' left SLF are illustrated (blue). The red cluster represents the overlaid ROI (Figure 3) due to the thresholded probability map ( $p<0.05$ ), whereas the golden cluster (see also Figure 5B) represents voxels of FA values $(-28,-28,44)$ that are significantly correlated $\left(r=0.758^{* *}\right)$ with the error rates of APs with respect to the AP-test. et al., 2002) that compared compact white matter (corpus callosum, interne capsule, cerebral peduncle) with non-compact white matter (corona radiata and peripheral white matter). These authors suggested that myelination is initially greater in compact white matter, whereas the change in myelination may be greater in non-compact white matter during early childhood. Moreover, our data lead us to suggest that a specific local anisotropic lateralization, characterizing the early matured compact core of the SLF, underpins the development of AP under favourable environmental conditions, such as an early start to musical training and deliberate practice (Baharloo et al., 1998; Vitouch, 2003).

Alternatively, we cannot rule out the possibility that the demonstrated anisotropic lateralization effects even in the core arcuate structure are due to plastic changes as a function of deliberate practice and musical expertise. But it is plausible to assume on the basis of our considerations and argumentation that effects of core white matter anisotropic characteristics more likely reflect the impact of earlier ontogenetic stages of development than do peripheral group-specific alterations.

On might be concerned about the strength of the statistical effects. Certainly it would have been desirable to investigate larger sample of musicians and NM, to end up with more robust statistical effects. However, given the rare incidence of AP musicians in the population the present sample size ( $n=13$ per group) should by all means considered a reasonable and representative number.

Second, since our study emphasizes a relationship between differences in structural architecture as a function of musical expertise and its implications for auditory and speech functions it would have been wishful to compare brain imaging data with behavioural data obtained from measurements of language proficiency. However to the best of our knowledge a suitable behavioural screening that covers a broad range of speech and language functions does not exist.

\section{CONCLUSIONS}

In summarizing our results, we conclude first that musical expertise combined with AP leads to a left-sided lateralization of mean FAvalues among the core structures of the $\mathrm{AF}$, as identified on the basis of high inter-individual morphological congruency. This effect is modulated considerably by the level of musical expertise (significant interaction musical expertise $\times$ hemisphere), since RP musicians revealed no inter-hemispheric differences. Obviously, the anisotropic properties of the selected SLF section represent a marked discriminatory feature of musical expertise and AP respectively.

Secondly, we identified three distinctive clusters exclusively in the left SLF in AP musicians, whose FA-values correlated positively with the error rate evaluated by the AP-test. This proficiency effect was not present in RP musicians. In other words, the higher the AP acuity in AP musicians the lower are the FA values within the identified three regions, two of which (Figures 5C,D) play an prominent role in language processing (Glasser and Rilling, 2008).

Thirdly, the Pioneer Axon Thesis proposes that peripheral white matter development (contrary to compact white matter core regions) is influenced considerably by environmental factors, in this case musical training, over a long period in postnatal life. Thus, deliberate music practice, which is the sine qua non of the acuity of AP, might be quantifiable in the form of particular alterations in white matter plasticity of auditory-related fasciculi like the SLF. 
With respect to the long-lasting debate as to whether AP is acquired or genetically disposed, further DTI studies would facilitate the investigation of familial aggregation of anisotropic characteristics within the early developing region of the arcuate core. If our suggestion holds true, a musician's family with many AP members should be separable from a musician's family without AP members. In fact, such an approach might provide a better understanding of the interaction of genetics and environmental factors that contribute to the manifestation of AP.

\section{REFERENCES}

Aboitiz, F., and Garcia, R. (1997). The anatomy of language revisited. Biol. Res. 30, 171-183.

Aboitiz, F., and Garcia, R. (2009). Merging of phonological and gestural circuits in early language evolution. Rev. Neurosci. 20, 71-84.

Alexander, A. L., Lee, J. E., Lazar, M., and Field, A. S. (2007). Diffusion tensor imaging of the brain. Neurotherapeutics 4, 316-329.

Annett, M. (1967). The binomial distribution of right, mixed and left handedness. Q. J. Exp. Psychol. 19, 327-333.

Ashtari, M., Cervellione, K. L., Hasan, K. M., Wu, J., McIlree, C., Kester, H., Ardekani, B. A., Roofeh, D., Szeszko, P. R., and Kumra, S. (2007). White matter development during late adolescence in healthy males: a cross-sectional diffusion tensor imaging study. Neuroimage 35, 501-510.

Baharloo, S., Johnston, P.A., Service, S. K., Gitschier, J., and Freimer, N. B. (1998). Absolute pitch: an approach for identification of genetic and nongenetic components. Am. J. Hum. Genet. 62, 224-231.

Bangert, M., Peschel, T., Schlaug, G., Rotte, M., Drescher, D., Hinrichs, H., Heinze, H. J., and Altenmuller, E. (2006). Shared networks for auditory and motor processing in professional pianists: evidence from fMRI conjunction. Neuroimage 30, 917-926.

Barnea-Goraly, N., Menon, V., Eckert, M., Tamm, L., Bammer, R., Karchemskiy, A., Dant, C. C., and Reiss, A. L. (2005). White matter development during childhood and adolescence: a cross-sectional diffusion tensor imaging study. Cereb. Cortex 15, 1848-1854.

Barrick, T. R., Lawes, I. N., Mackay, C. E., and Clark, C. A. (2007). White matter pathway asymmetry underlies functional lateralization. Cereb. Cortex 17, 591-598.

Bartzokis, G., Beckson, M., Lu, P. H., Nuechterlein, K. H., Edwards, N., and Mintz, J. (2001). Age-related changes in frontal and temporal lobe volumes in men: a magnetic resonance imaging study. Arch. Gen. Psychiatry 58, 461-465.
Baumann, S., Koeneke, S., Schmidt, C. F., Meyer, M., Lutz, K., and Jäncke, L. (2007). A network for audio-motor coordination in skilled pianists and nonmusicians. Brain Res. 1161, 65-78.

Baumann, S., Meyer, M., and Jäncke, L. (2008). Enhancement of auditoryevoked potentials in musicians reflects an influence of expertise but not selective attention. J. Cogn. Neurosci. 20 , 2238-2249.

Beaulieu, C. (2002). The basis of anisotropic water diffusion in the nervous system - a technical review. NMR Biomed. 15, 435-455.

Beaulieu, C., Plewes, C., Paulson, L.A., Roy, D., Snook, L., Concha, L., and Phillips, L. (2005). Imaging brain connectivity in children with diverse reading ability. Neuroimage 25, 1266-1271.

Bengtsson, S. L., Nagy, Z., Skare, S., Forsman, L., Forssberg, H., and Ullen, F. (2005). Extensive piano practicing has regionally specific effects on white matter development. Nat. Neurosci. 8, 1148-1150.

Bermudez, P., Lerch, J. P., Evans, A. C., and Zatorre, R. J. (2009). Neuroanatomical correlates of musicianship as revealed by cortical thickness and voxel-based 1583-1596.

Besson, M., Schon, D., Moreno, S., Santos, A., and Magne, C. (2007). Influence of musical expertise and musical training on pitch processing in music and language. Restor. Neurol. Neurosci. 25 , 399-410.

Bhagat, Y. A., and Beaulieu, C. (2004). Diffusion anisotropy in subcortical white matter and cortical gray matter: changes with aging and the role of CSF-suppression. J. Magn. Reson. Imaging 20, 216-227.

Buchsbaum, B., Hickok, G., and Humphries, C. (2001). Role of left posterior superior temporal gyrus in phonological processing for speech perception and production. Cogn. Sci. 25, 663-678.

Cascio, C. J., Gerig, G., and Piven, J. (2007). Diffusion tensor imaging: application to the study of the developing brain. J. Am. Acad. Child Adolesc. Psychiatry 46, 213-223.

Catani, M., Allin, M. P., Husain, M., Pugliese, L., Mesulam, M. M., Murray, morphometry. Cereb. Cortex 19,

\section{ACKNOWLEDGMENTS}

We are indebted to Nicki Langer for assisting in MR data acquisition, Jürgen Hänggi for methodological advice, and Marcus Cheetham for comments on the earlier version of the manuscript. Funding was provided by Swiss National Foundation (SNF 46234101 to Lutz Jäncke and Mathias S. Oechslin, SNF 320000-120660-1 to Martin Meyer) and Research funding of the University of Zurich (56234101 to Mathias S. Oechslin).

R. M., and Jones, D. K. (2007) Symmetries in human brain language pathways correlate with verbal recall. Proc. Natl. Acad. Sci. U.S.A. 104 17163-17168.

Catani, M., and Mesulam, M. (2008). The arcuate fasciculus and the disconnection theme in language and aphasia: history and current state. Cortex 44 953-961.

Dubois, J., Dehaene-Lambertz, G. Perrin, M., Mangin, J. F., Cointepas, Y., Duchesnay, E., Le Bihan, D., and Hertz-Pannier, L. (2008). Asynchrony of the early maturation of white matter bundles in healthy infants: quantitative landmarks revealed noninvasively by diffusion tensor imaging. Hum. Brain Mapp. 29, 14-27.

Elbert, T., Pantev, C., Wienbruch, C. Rockstroh, B., and Taub, E. (1995). Increased cortical representation of the fingers of the left hand in string players. Science 270, 305-307.

Eluvathingal, T. J., Hasan, K. M., Kramer, L., Fletcher, J. M., and Ewing-Cobbs, L. (2007). Quantitative diffusion tensor tractography of association and projection fibers in normally developing children and adolescents. Cereb. Cortex 17, 2760-2768.

Frey, S., Campbell, J. S., Pike, G. B., and Petrides, M. (2008). Dissociating the human language pathways with high angular resolution diffusion fiber tractography. J. Neurosci. 28, 11435-11444

Friederici, A. D. (2009). Allocating functions to fiber tracts: facing its indirectness. Trends Cogn. Sci. 13 370-371.

Fujioka, T., Ross, B., Kakigi, R., Pantev, C., and Trainor, L. J. (2006). One year of musical training affects development of auditory cortical-evoked fields in young children. Brain 129, 2593-2608.

Fujioka, T., Trainor, L. J., Ross, B., Kakigi, R., and Pantev, C. (2004). Musical training enhances automatic encoding of melodic contour and interval structure. J. Cogn. Neurosci. 16, 1010-1021.

Fujioka, T., Trainor, L. J., Ross, B., Kakigi, R., and Pantev, C. (2005). Automatic encoding of polyphonic melodies in musicians and nonmusicians. J. Cogn. Neurosci. 17, 1578-1592.
Gaab, N., Schulze, K., Ozdemir, E., and Schlaug, G. (2006). Neural correlates of absolute pitch differ between blind and sighted musicians. Neuroreport 17 , 1853-1857.

Gao, W., Lin, W., Chen, Y., Gerig, G., Smith, J. K., Jewells, V., and Gilmore, J. H. (2009). Temporal and spatial development of axonal maturation and myelination of white matter in the developing brain. AJNR Am. J. Neuroradiol. 30, 290-296.

Gaser, C., and Schlaug, G. (2003). Brain structures differ between musicians and non-musicians. J. Neurosci. 23, 9240-9245.

Geschwind, N. (1970). The organization of language and the brain. Science 170, 940-944.

Glasser, M.F., and Rilling, J. K. (2008). DTI tractography of the human brain's language pathways. Cereb. Cortex 18, 2471-2482.

Gogtay, N., Giedd, J. N., Lusk, L., Hayashi, K. M., Greenstein, D., Vaituzis, A. C., Nugent, T. F. III, Herman, D. H., Clasen, L. S., Toga, A. W., Rapoport, J. L., and Thompson, P. M. (2004). Dynamic mapping of human cortical development during childhood through early adulthood. Proc. Natl. Acad. Sci. U.S.A. 101, 8174-8179.

Gold, B. T., Powell, D. K., Xuan, L., Jiang, Y., and Hardy, P. A. (2007). Speed of lexical decision correlates with diffusion anisotropy in left parietal and frontal white matter: evidence from diffusion tensor imaging. Neuropsychologia 45 , 2439-2446.

Guan, K. L., and Rao, Y. (2003). Signalling mechanisms mediating neuronal responses to guidance cues. Nat. Rev Neurosci. 4, 941-956.

Hagmann, P., Cammoun, L., Martuzzi, R., Maeder, P., Clarke, S., Thiran, J. P., and Meuli, R. (2006a). Hand preference and sex shape the architecture of language networks. Hum. Brain Mapp. 27, 828-835.

Hagmann, P., Jonasson, L., Maeder, P., Thiran, J. P., Wedeen, V. J., and Meuli, R. (2006b). Understanding diffusion MR imaging techniques: from scalar diffusion-weighted imaging to diffusion tensor imaging and beyond. Radiographics 26(Suppl. 1), S205-223. 
Hänggi, J., Beeli, G., Oechslin, M. S., and Jäncke, L. (2008). The multiple synaesthete E.S.: neuroanatomical basis of interval-taste and tone-colour synaesthesia. Neuroimage 43, 192-203.

Herholz, S. C., Lappe, C., and Pantev, C. (2009). Looking for a pattern: an MEG study on the abstract mismatch negativity in musicians and nonmusicians. BMC Neurosci. 10, 42.

Hickok, G., and Poeppel, D. (2007). The cortical organization of speech processing. Nat. Rev. Neurosci. 8, 393-402.

Hutchinson, S., Lee, L. H., Gaab, N., and Schlaug, G. (2003). Cerebellar volume of musicians. Cereb. Cortex 13, 943-949.

Imfeld, A., Oechslin, M. S., Meyer, M., Loenneker, T., and Jäncke, L. (2009). White matter plasticity in the corticospinal tract of musicians: a diffusion tensor imaging study. Neuroimage 46, 600-607.

Jäncke, L., Baumann, S., Koeneke, S., Meyer, M., Laeng, B., Peters, M., and Lutz, K. (2006). Neural control of playing a reversed piano: empirical evidence for an unusual cortical organization of musical functions. Neuroreport 17, 447-451.

Jäncke, L., Shah, N. J., and Peters, M. (2000). Cortical activations in primary and secondary motor areas for complex bimanual movements in professional pianists. Brain Res. Cogn. Brain Res. 10, 177-183.

Jiang, H., van Zijl, P. C., Kim, J., Pearlson, G. D., and Mori, S. (2006). DtiStudio: resource program for diffusion tensor computation and fiber bundle tracking. Comput. Methods Programs Biomed. 81, 106-116.

Keenan, J.P., Thangaraj, V., Halpern, A. R., and Schlaug, G. (2001). Absolute pitch and planum temporale. Neuroimage 14, 1402-1408.

Klingberg, T., Hedehus, M., Temple, E., Salz, T., Gabrieli, J. D., Moseley, M. E., and Poldrack, R. A. (2000). Microstructure of temporo-parietal white matter as a basis for reading ability: evidence from diffusion tensor magnetic resonance imaging. Neuron 25, 493-500.

Koeneke,S.,Lutz, K., Herwig, U.,Ziemann, U., and Jäncke, L. (2006). Extensive training of elementary finger tapping movements changes the pattern of motor cortex excitability. Exp. Brain Res. 174, 199-209.

Kreher, B. W., Hennig, J., and Il'yasov, K. A. (2006). DTI\&FiberTools: a complete toolbox for DTI calculation, fiber tracking, and combined evaluation. In Proceeding of ISMRM 14th International Scientific Meeting, Seattle, USA.
Kuhl, P. K. (2004). Early language acquisition: cracking the speech code. Nat. Rev. Neurosci. 5, 831-843.

Lahav, A., Saltzman, E., and Schlaug, G. (2007). Action representation of sound: audiomotor recognition network while listening to newly acquired actions. J. Neurosci. 27, 308-314.

LaMantia,A.S., and Rakic, P. (1990). Axon overproduction and elimination in the corpus callosum of the developing rhesus monkey. J. Neurosci. 10, 2156-2175.

Lappe, C., Herholz, S. C., Trainor, L. J., and Pantev, C. (2008). Cortical plasticity induced by short-term unimodal and multimodal musical training. $J$. Neurosci. 28, 9632-9639.

Levitin, D. J., and Rogers, S. E. (2005). Absolute pitch: perception, coding, and controversies. Trends Cogn. Sci. 9, 26-33.

Lotze, M., Scheler, G., Tan, H. R., Braun, C., and Birbaumer, N. (2003). The musician's brain: functional imaging of amateurs and professionals during performance and imagery. Neuroimage 20, 1817-1829.

Lüders, E., Gaser, C., Jäncke, L., and Schlaug, G. (2004). A voxel-based approach to gray matter asymmetries. Neuroimage 22, 656-664.

Makris, N., Kennedy, D. N., McInerney, S., Sorensen, A. G., Wang, R., Caviness, V. S. Jr., and Pandya, D. N. (2005). Segmentation of subcomponents within the superior longitudinal fascicle in humans: a quantitative, in vivo, DT-MRI study. Cereb. Cortex 15, 854-869.

Makris, N., Papadimitriou, G. M., Kaiser, J. R.,Sorg, S., Kennedy, D. N., and Pandya, D. N. (2009). Delineation of the middle longitudinal fascicle in humans: a quantitative, in vivo, DT-MRI study. Cereb. Cortex 19, 777-785.

Marques, C., Moreno, S., Castro, S. L., and Besson, M. (2007). Musicians detect pitch violation in a foreign language better than nonmusicians: behavioral and electrophysiological evidence. $J$. Cogn. Neurosci. 19, 1453-1463.

McGraw, P., Liang, L., and Provenzale, J. M. (2002). Evaluation of normal agerelated changes in anisotropy during infancy and childhood as shown by diffusion tensor imaging. AJR Am. J. Roentgenol. 179, 1515-1522.

Moreno, S., Marques, C., Santos, A., Santos, M., Castro, S. L., and Besson, M. (2009). Musical training influences linguistic abilities in 8-year-old children: more evidence for brain plasticity. Cereb. Cortex 19, 712-723.

Mori, S., Crain, B. J., Chacko, V.P., and van Zijl, P. C. (1999). Three-dimensional tracking of axonal projections in the brain by magnetic resonance imaging. Ann. Neurol. 45, 265-269.
Mori, S., Kaufmann, W.E., Davatzikos, C., Stieltjes, B., Amodei, L., Fredericksen, K., Pearlson, G. D., Melhem, E. R. Solaiyappan, M., Raymond, G. V., Moser, H.W., and van Zijl, P.C. (2002). Imaging cortical association tracts in the human brain using diffusiontensor-based axonal tracking. Magn. Reson. Med. 47, 215-223.

Mori, S., and Zhang, J. (2006). Principles of diffusion tensor imaging and its applications to basic neuroscience research. Neuron 51, 527-539.

Moseley, M. (2002). Diffusion tensor imaging and aging - a review. NMR. Biomed. 15, 553-560.

Mukherjee, P., Miller, J. H., Shimony, J. S., Philip, J. V., Nehra, D., Snyder, A. Z., Conturo, T. E., Neil, J. J., and McKinstry, R. C. (2002). Diffusiontensor MR imaging of gray and white matter development during normal human brain maturation. AJNR Am. J. Neuroradiol. 23, 1445-1456.

Münte, T. F., Altenmüller, E., and Jäncke, L. (2002). The musician's brain as a model of neuroplasticity. Nat. Rev. Neurosci. 3, 473-478.

Musacchia, G., Sams, M., Skoe, E., and Kraus, N. (2007). Musicians have enhanced subcortical auditory and audiovisual processing of speech and music.Proc. Natl. Acad. Sci. U.S.A. 104, 15894-15898.

Niogi, S. N., and McCandliss, B. D. (2006). Left lateralized white matter microstructure accounts for individual differences in reading ability and disability. Neuropsychologia 44 , 2178-2188.

Oechslin, M. S., Meyer, M., and Jäncke, L. (2010). Absolute pitch - functional evidence of speech-relevant auditory acuity. Cereb. Cortex. 20, 447-455.

Ohnishi, T., Matsuda, H.,Asada, T., Aruga, M., Hirakata, M., Nishikawa, M., Katoh, A., and Imabayashi, E. (2001). Functional anatomy of musical perception in musicians. Cereb. Cortex 11,754-760.

Pantev, C., Engelien, A., Candia, V., and Elbert, T. (2001). Representational cortex in musicians. Plastic alterations in response to musical practice. Ann. N. Y. Acad. Sci. 930, 300-314.

Pantev, C., Oostenveld, R., Engelien, A., Ross, B., Roberts, L. E., and Hoke, M. (1998). Increased auditory cortical representation in musicians. Nature 392, 811-814.

Partridge, S. C., Mukherjee, P., Berman, J. I., Henry, R. G., Miller, S. P., Lu, Y., Glenn, O. A., Ferriero, D. M., Barkovich, A. J., and Vigneron, D. B. (2005). Tractography-based quantitation of diffusion tensor imaging parameters in white matter tracts of preterm newborns. J. Magn. Reson. Imaging 22, 467-474.
Partridge, S. C., Mukherjee, P., Henry, R. G., Miller, S. P., Berman, J. I., Jin, H., Lu, Y., Glenn, O. A., Ferriero, D. M., Barkovich, A. J., and Vigneron, D. B. (2004). Diffusion tensor imaging: serial quantitation of white matter tract maturity in premature newborns. Neuroimage 22, 1302-1314.

Paus, T. (2005). Mapping brain maturation and cognitive development during adolescence. Trends Cogn. Sci. 9, 60-68.

Paus, T., Zijdenbos, A., Worsley, K., Collins, D. L., Blumenthal, J., Giedd, J. N., Rapoport, J. L., and Evans, A. C. (1999). Structural maturation of neural pathways in children and adolescents: in vivo study. Science 283 , 1908-1911.

Peretz, I., and Zatorre, R. J. (2005). Brain organization for music processing Annu. Rev. Psychol. 56, 89-114.

Pujol, J., Soriano-Mas, C., Ortiz, H., Sebastian-Galles, N., Losilla, J. M., and Deus, J. (2006). Myelination of language-related areas in the developing brain. Neurology 66, 339-343.

Rilling, J. K., Glasser, M. F., Preuss, T. M., Ma, X., Zhao, T., Hu, X., and Behrens, T.E. (2008). The evolution of the arcuate fasciculus revealed with comparative DTI. Nat. Neurosci. 11, 426-428.

Schlaug, G., Jäncke, L., Huang, Y., Staiger, J. F., and Steinmetz,H. (1995a). Increased corpus callosum size in musicians. Neuropsychologia 33, 1047-1055.

Schlaug, G., Jäncke, L., Huang, Y., and Steinmetz, H. (1995b). In vivo evidence of structural brain asymmetry in musicians. Science 267, 699-701.

Schmithorst, V. J., and Wilke, M. (2002). Differences in white matter architecture between musicians and nonmusicians: a diffusion tensor imaging study. Neurosci. Lett. 321, 57-60.

Schmithorst, V. J., Wilke, M., Dardzinski, B. J., and Holland, S. K. (2002). Correlation of white matter diffusivity and anisotropy with age during childhood and adolescence: a crosssectional diffusion-tensor MR imaging study. Radiology 222, 212-218.

Schneider, J. F., Il'yasov, K. A., Hennig, J., and Martin, E. (2004). Fast quantitative diffusion-tensor imaging of cerebral white matter from the neonatal period to adolescence. Neuroradiology 46, 258-266.

Schneider, P., Andermann, M., Wengenroth, M., Goebel, R., Flor, H., Rupp, A., and Diesch, E. (2009). Reduced volume of Heschl's gyrus in tinnitus. Neuroimage 45, 927-939.

Schneider, P., Scherg, M., Dosch, H. G., Specht, H. J., Gutschalk, A., and Rupp, A. (2002). Morphology of Heschl's gyrus reflects enhanced activation in the auditory cortex of musicians. Nat. Neurosci. 5, 688-694. 
Schneider, P., Sluming, V., Roberts, N., Scherg, M., Goebel, R., Specht, H. J., Dosch, H. G., Bleeck, S., Stippich, C., and Rupp, A. (2005). Structural and functional asymmetry of lateral Heschl's gyrus reflects pitch perception preference. Nat. Neurosci. 8, 1241-1247.

Schön, D., Magne, C., and Besson, M. (2004). The music of speech: music training facilitates pitch processing in both music and language. Psychophysiology 41, 341-349.

Schulz, M., Ross, B., and Pantev, C. (2003). Evidence for training-induced crossmodal reorganization of cortical functions in trumpet players. Neuroreport $14,157-161$.

Shahin, A., Roberts, L. E., Pantev, C., Trainor, L. J., and Ross, B. (2005). Modulation of P2 auditory-evoked responses by the spectral complexity of musical sounds. Neuroreport 16, 1781-1785.

Shahin, A. J., Roberts, L. E., Chau, W., Trainor, L. J., and Miller, L. M. (2008). Music training leads to the development of timbre-specific gamma band activity. Neuroimage 41, 113-122.

Sluming, V., Barrick, T., Howard, M., Cezayirli, E., Mayes, A., and Roberts, N. (2002). Voxel-based morphometry reveals increased gray matter density in Broca's area in male symphony orchestra musicians. Neuroimage 17, 1613-1622.
Sluming, V., Brooks, J., Howard, M., Downes, J. J., and Roberts, N. (2007). Broca's area supports enhanced visuospatial cognition in orchestral musicians. J. Neurosci. 27, 3799-3806.

Snook, L., Paulson, L. A., Roy, D., Phillips, L., and Beaulieu, C. (2005). Diffusion tensor imaging of neurodevelopment in children and young adults. Neuroimage 26, 1164-1173.

Snook, L., Plewes, C., and Beaulieu, C. (2007). Voxel based versus region of interest analysis in diffusion tensor imaging of neurodevelopment. Neuroimage 34, 243-252.

Sperry, R.W. (1963). Chemoaffinity in the orderly growth of nerve fiber patterns and connections. Proc. Natl. Acad. Sci. U.S.A. 50, 703-710.

Su, P., Kuan, C. C., Kaga, K., Sano, M., and Mima, K. (2008). Myelination progression in language-correlated regions in brain of normal children determined by quantitative MRI assessment. Int. J. Pediatr. Otorhinolaryngol. 72, 1751-1763.

Sundgren, P. C., Dong, Q., GomezHassan, D., Mukherji, S. K., Maly, P., and Welsh, R. (2004). Diffusion tensor imaging of the brain: review of clinical applications. Neuroradiology 46, 339-350.

Takahashi, M., Ono, J., Harada, K., Maeda, M., and Hackney, D. B.
(2000). Diffusional anisotropy in cranial nerves with maturation: quantitative evaluation with diffusion MR imaging in rats. Radiology 216, 881-885.

Tessier-Lavigne, M., and Goodman, C. S. (1996). The molecular biology of axon guidance. Science 274, 1123-1133.

Upadhyay, J., Silver, A., Knaus, T. A., Lindgren, K. A., Ducros, M., Kim, D. S., and Tager-Flusberg, H. (2008). Effective and structural connectivity in the human auditory cortex. J. Neurosci. 28, 3341-3349.

Vigneau, M., Beaucousin, V., Herve, P. Y., Duffau, H., Crivello, F., Houde, O. Mazoyer, B., and Tzourio-Mazoyer N. (2006). Meta-analyzing left hemisphere language areas: phonology, semantics, and sentence processing. Neuroimage 30, 1414-1432.

Vitouch, O. (2003). Absolutist models of absolute pitch are absolutely misleading. Music Percept. 21, 111-117.

Wrigley, P. J., Gustin, S. M., Macey, P. M., Nash, P.G., Gandevia, S. C., Macefield, V.G., Siddall, P.J., and Henderson, L.A (2009).Anatomical changes in human motor cortex and motor pathways following complete thoracic spinal cord injury. Cereb. Cortex. 19, 224-232.

Yu, T. W., and Bargmann, C. I. (2001). Dynamic regulation of axon guidance. Nat. Neurosci. 4(Suppl.), 1169-1176.
Zhang, J., Evans, A., Hermoye, L., Lee, S. K., Wakana, S., Zhang, W., Donohue, P., Miller, M. I., Huang, H., Wang, X., van Zijl, P. C., and Mori, S. (2007) Evidence of slow maturation of the superior longitudinal fasciculus in early childhood by diffusion tensor imaging. Neuroimage 38, 239-247.

Conflict of Interest Statement: The authors declare that the research was conducted in the absence of any commercial or financial relationships that could be construed as a potential conflict of interest.

Received: 16 September 2009; paper pending published: 13 October 2009; accepted: 23 December 2009; published online: 08 February 2010.

Citation: Oechslin MS, Imfeld A, Loenneker T, Meyer M and Jäncke L (2010) The plasticity of the superior longitudinal fasciculus as a function of musical expertise: a diffusion tensor imaging study. Front. Hum. Neurosci. 3:76. doi: 10.3389/neuro.09.076.2009

Copyright (c) 2010 Oechslin, Imfeld, Loenneker, Meyer and Jäncke. This is an open-access article subject to an exclusive license agreement between the authors and the Frontiers Research Foundation, which permits unrestricted use, distribution, and reproduction in any medium, provided the original authors and source are credited. 